\title{
Un estudio analiza distintos modelos de recuperación de pueblos abandonados en el Alto Aragón
}

En 2017 se celebró en Huesca el II Congreso Nacional contra la Despoblación, donde se impartieron diversos talleres temáticos con el propósito de analizar el problema desde distintas perspectivas. En el taller dedicado al área de Urbanismo y la Ordenación del Territorio se mostraron varias iniciativas de recuperación de asentamientos, así como diferentes estrategias para la puesta en valor del medio rural en su conjunto. A raíz de esta experiencia, la Diputación Provincial de Huesca encargó a Sixto Marín Gavín, arquitecto coordinador del citado taller, la redacción de un estudio sobre distintas experiencias de pueblos altoaragoneses recuperados desde los años 90 del siglo XX. Sobre la base de la información recogida por este para su tesis, y con la incorporación de profesionales de otras disciplinas, se completó en diciembre de 2017 un documento para dar a conocer de manera sistemática y detallada la realidad de este fenómeno.

Sixto Marín Gavín | arquitecto

URL de la contribución <www.iaph.es/revistaph/index.php/revistaph/article/view/4430>

\section{La despoblación en el Alto Aragón}

El territorio del Alto Aragón tiene una larga tradición tanto en el abandono como en la recuperación de núcleos rurales, especialmente en zonas de montaña, tanto del Pirineo como de las sierras. Estos son territorios en los que las condiciones económicas han sido históricamente difíciles, basadas en una economía de subsistencia dependiente de la agricultura, la ganadería y de pequeñas industrias y artesanías. Muchos de estos núcleos estaban en lugares de difícil acceso, alejados de las vías principales, en fondos de valles o laderas que dificultaban los intercambios comerciales y las comunicaciones con las localidades principales, que impedían el acceso a determinados servicios que cada vez se hacían más imprescindibles, como la educación.

Paralelamente, sobre todo a partir de los años 60 , se dieron además dos fenómenos socioeconómicos que, si bien no fueron determinantes en sí mismos, es bien cierto que marcaron el carácter de algunos de los procesos de despoblación. Por un lado, la incipiente industrialización del país, que atrajo a las ciudades a gran cantidad de personas con la promesa de mejores condiciones de vida. Por otro, la construcción de determinados embalses y los procesos de reforestación de montes, vinculados a estos, y que supusieron la expropiación de tierras

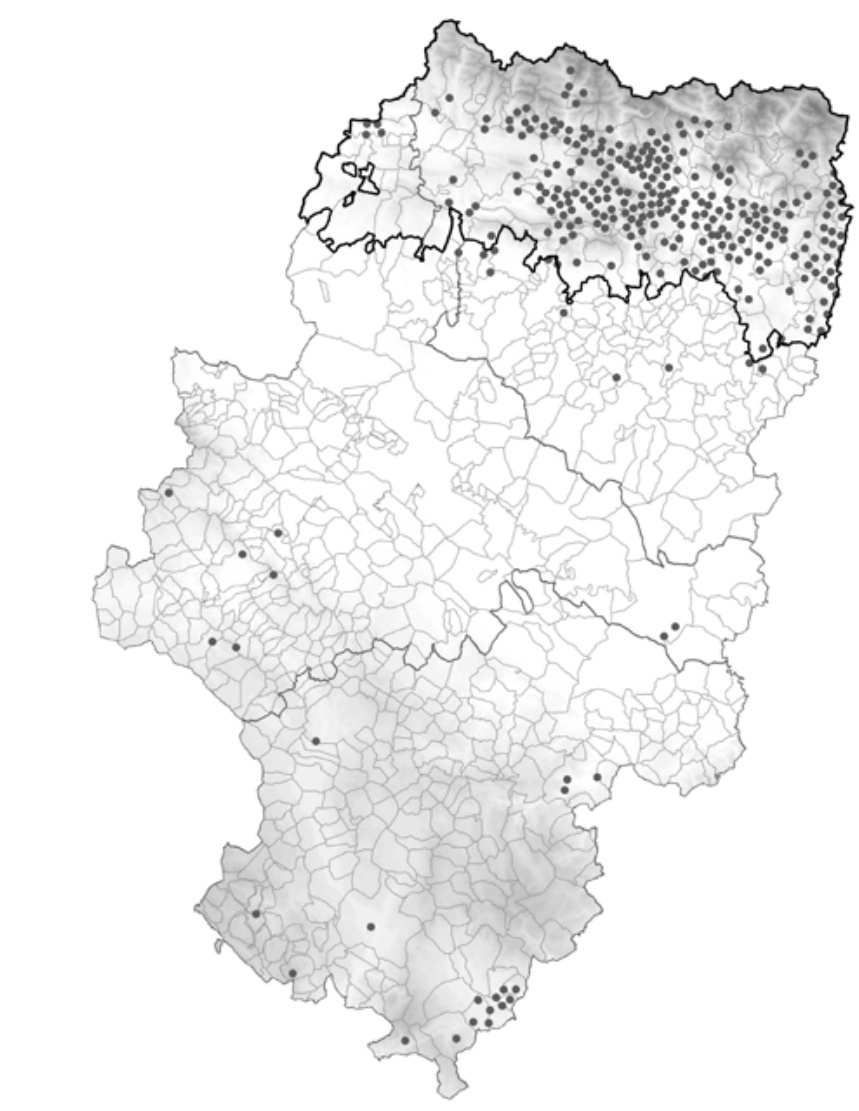

Mapa de Aragón con la situación de los asentamientos despoblados en los años 80 del siglo XX | fuente ACÍN FANLO; PINILLA NAVARRO, 1995 
con la consiguiente emigración de sus habitantes. Todo esto ocurrió desde los años 30 hasta los 80 del siglo $X X$ y el resultado fue un conjunto de más de 200 núcleos despoblados a lo largo de la geografía aragonesa.

\section{La recuperación de despoblados en el Alto Aragón} Sin embargo, a principios de la década de 1980, el deseo de recuperación de estos lugares, de volver a ellos como una necesidad de recuperar las raíces personales que se iban perdiendo en la ciudad, hizo que algunos núcleos despoblados fueran retomando la vida. Aunque estas iniciativas se producían indistintamente en pueblos de propiedad pública o privada, la mayor parte del conjunto de pueblos abandonados en el Alto Aragón pertenecían al Estado. La intervención en ellos resultaba complicada ya que no se preveía la reversión a sus antiguos propietarios y era complicado gestionar su recuperación para nuevos usos.

Estas experiencias no han formado parte de una estrategia territorial de conservación del patrimonio como tal, sino que han sido más bien un conjunto de trabajos heterogéneos, dispersos y con resultados bien distintos. La sistematización en su análisis permite establecer paralelismos y extraer conclusiones sobre los que trazar estrategias a medio/largo plazo para la revitalización de los asentamientos despoblados en su conjunto, poniendo así en valor los paisajes culturales de los que son protagonistas.

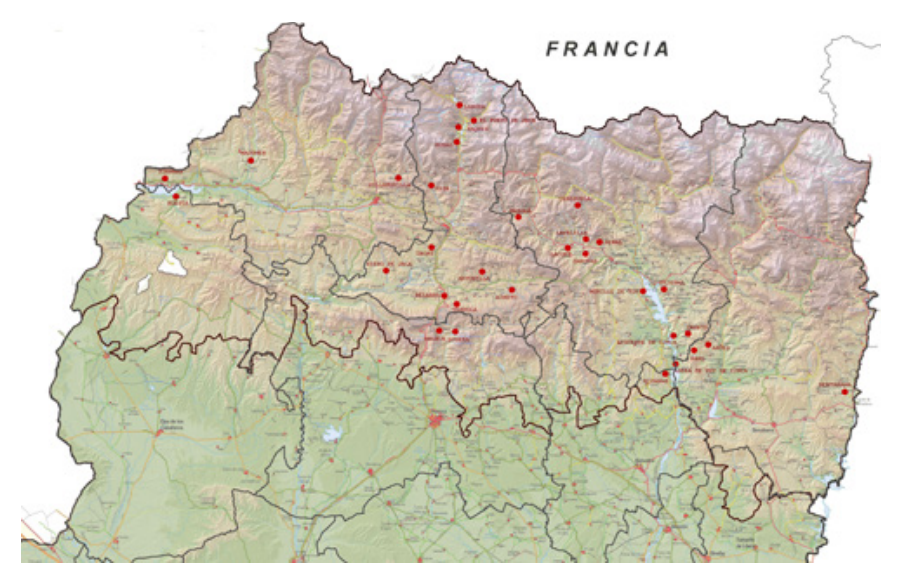

Marco del estudio. Los repoblados sobre plano hipsométrico. Relación de los asentamientos con el territorio | fuente Elaboración propia
Existen distintas formas de agrupar el conjunto de las iniciativas, desde su titularidad a su localización pero, para el estudio de su transformación, nos parecía más interesante el análisis del uso final al que se han dedicado. De forma genérica los podemos clasificar en habitacionales, vacacionales, sociales y educativos.

\section{$>$ Habitacionales}

En esta tipología incluimos los pueblos que se han recuperado con la finalidad de ser habitados ya sea como primera o segunda residencia, intentando además dotarlos de actividad económica basada no solo en el trabajo de la tierra y de la ganadería, sino también en el establecimiento de diversos talleres, artesanías o turismo.

En primer lugar tenemos los núcleos de titularidad privada recuperados por antiguos pobladores o nuevos propietarios. Entre los mismos destacaríamos Majones, Lanuza, Villanovilla, Belarra, Yespola, Belsue, Lusera, Bergua, Janovas o Montañana. El esfuerzo dedicado a su recuperación corresponde a sus habitantes y, en la mayor parte de los casos, son usados como segunda residencia. La rehabilitación se ha producido siguiendo la normativa y con respeto a la arquitectura tradicional. Excepto Janovas, todos ellos han sido rehabilitados en la década de los 90 y la recuperación y conservación tanto de las edificaciones como del entorno es excepcional. Janovas se encuentra inmerso en un proceso de reversión pero ya es visible la mejora en algunas edificaciones y la urbanización del núcleo.

Por otro lado tenemos el conjunto de núcleos de propiedad pública que han sido cedidos a asociaciones para su recuperación y disfrute por un periodo que va de los 25 a los 50 años. Quienes promueven este modelo provienen de la ciudad; hombres y mujeres jóvenes, normalmente sin relación vital alguna con el lugar que van a recuperar, y que se inscriben dentro del movimiento que se conoce como neorrurales.

Los ejemplos paradigmáticos en el Alto Aragón son Aineto, Artosilla e Ibort que juntos conforman una asociación para defender sus derechos que se llama 


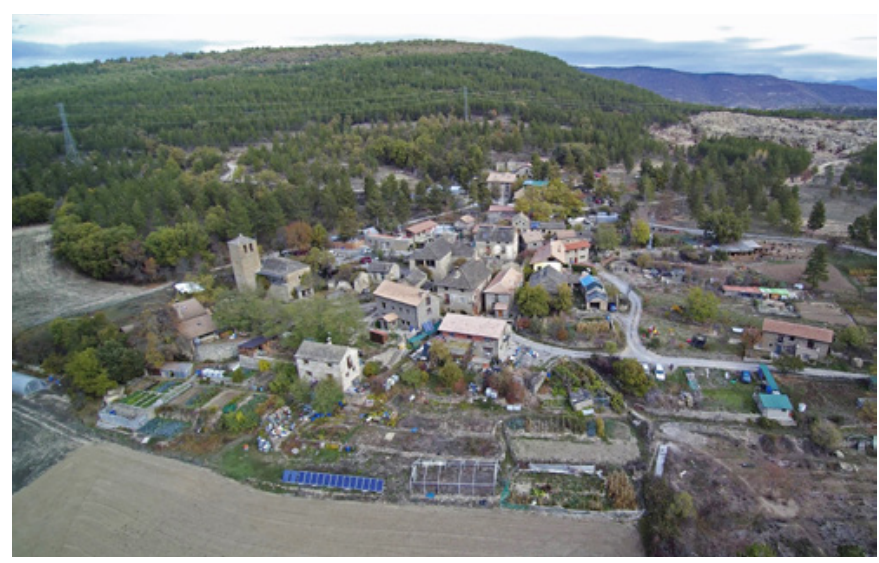

Ibort (Sabiñánigo, Huesca). Pueblo recuperado por Artoborain | foto Sixto Marín

Artiborain. Existen otros como Caneto (Asociación la Senda) o Mipanas (cooperativa la Bitolosa) que, aunque de manera autónoma, han conseguido resultados reseñables en sus respectivos asentamientos. Este conjunto de pobladores fueron pioneros e iniciaron sus respectivos procesos a mediados de los 80. Excepto Mipanas, que está al $80 \%$, el resto ya ha recuperado la totalidad de los asentamientos y están proyectando ampliaciones para atraer nuevos pobladores.

\section{$>$ Vacacionales}

El objetivo de la recuperación de estos núcleos es convertirse en un centro de vacaciones, un lugar abierto a todo el mundo, con una finalidad lúdica. El modelo de estos lugares enlaza con la idea de los centros de vacaciones ligados a empresas, sindicatos e incluso de algunas administraciones públicas.

En un primer grupo tendríamos a Ruesta (CGT), Morillo de Tou (UGT) y Ligüerre de Cinca (CCOO). Todos están ligados a la iniciativa de organizaciones sindicales. En todos los casos se ha intentado mantener las estructuras arquitectónicas originales y el trazado urbano que le dan personalidad al lugar y que, al mismo tiempo, los diferencian de otras ofertas turísticas. Tanto las rehabilitaciones como las edificaciones de nueva planta mantienen el espíritu de la arquitectura tradicional adaptada a las nuevas formas de construcción, lo que facilita su mantenimiento y garantiza la comodidad necesaria para quienes las van a usar.
También estos núcleos se rigen por un sistema de cesión, que no de propiedad, aunque con el paso del tiempo se va trabajando en la dirección de que dicha propiedad pase a los usufructuarios. Como en el caso de los pueblos cedidos a Artiborain y otras asociaciones, estos procesos se inician a mediados de los años $80 \mathrm{y}$, excepto Ruesta (al $10 \%)$, el resto están recuperados completamente. Estos centros vacacionales han ampliado su oferta implantando áreas de camping y otras instalaciones auxiliares, debidamente integradas en el contexto natural del que disfrutan.

Por otro lado tenemos iniciativas como la de Atiart que contempla la recuperación de un núcleo para centro vacacional pero que es de propiedad particular y está promovido por un empresario de la zona. Las directrices de recuperación han sido las mismas pero los objetivos son más modestos. El proceso de rehabilitación se inició a principios del 2010 y está a punto de finalizar.

El aspecto que destacamos de este modelo es la reconstrucción del lugar con un fin fundamentalmente turístico. Paralelamente, nos parece interesante el hecho de que se hayan puesto en cultivo tierras abandonadas con cultivos tradicionales de la zona y la realización de una manufactura, en este caso vino, que permita su comercialización.

\section{$>$ Sociales}

En este punto se incluirían aquellos núcleos que se rehabilitan con el fin primordial de dar servicio a colectivos especiales: fundamentalmente personas con diversidad funcional, ofreciéndoles estancias en el medio natural con una adaptación a sus necesidades.

En este grupo destaca la figura de Isín (Fundación Benito Ardid) que posee toda sus instalaciones adaptadas así como las habitaciones y las calles o accesos. El proceso de rehabilitación que se inició en el año 2000 está completamente acabado y, como ocurría con los anteriores, se han añadido a la estructura original nuevas instalaciones para mejorar la oferta.

El uso prioritario es para las personas pertenecientes a la fundación que se encarga de la rehabilitación de aquellas otras entidades con las que puedan existir acuerdos. 
Sin embargo, su viabilidad pasa por ofertar estancias al público en general siempre que haya plazas disponibles.

\section{$>$ Educativo}

El último uso de pueblos rehabilitados es el educativo. Este es un uso muy específico promovido por diversos ministerios (Educación, Fomento y Medio Ambiente o sus equivalentes) y gobiernos regionales o por asociaciones culturales.

La experiencia solo se desarrolla como iniciativa pública en Búbal mientras que por el lado privado se podrían destacar Griebal (Boy Scouts de Aragón), Pano (Fundación Pano) o Aldea de Puy de Cinca (UAGA). Mientras Bubal inicia su recuperación a mediados de los años 80 , el resto de asentamientos no empiezan a moverse hasta finales de los 90. Excepto el primero, el resto están todavía en proceso de recuperación, aunque son habitables parcialmente. La recuperación de todos ellos ha conllevado una mejora sustancial de sus entornos.

El proyecto en todos surge como una manera de acercar la vida rural tradicional a las y los escolares, especialmente a los que viven en la ciudad y tienen entre 14 y 18 años, que deseen conocer zonas distintas y convivir con estudiantes de otros lugares del país.

Las estancias son de periodos en torno a una semana y se adaptan a unos programas preestablecidos en los que se trabajan aspectos de rehabilitación de edificios, tareas

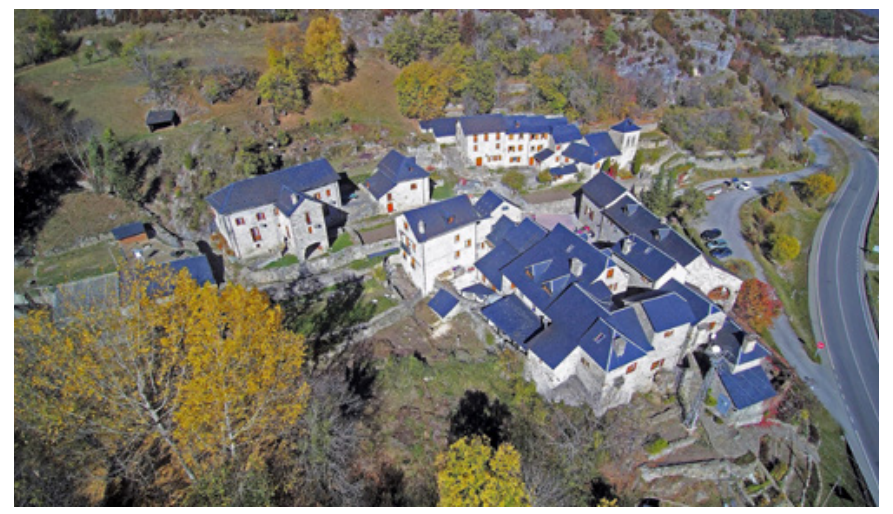

Bubal (Biescas, Huesca). Pueblo recuperado por el Gobierno | foto Sixto Marín agro-ganaderas, talleres de artesanía, conocimiento del medio natural, social y de las formas de vida tradicionales.

El uso educativo de estos lugares es el aspecto más destacado porque realiza una importante labor de divulgación de los modos de vida tradicionales. La ocupación es muy alta durante todo el año pero no genera directamente una aportación de riqueza a la zona y no hay posibilidades de uso privado de las instalaciones.

Los núcleos de montaña estudiados se han ido construyendo a lo largo de siglos, respondiendo de manera orgánica a las necesidades de sus ocupantes o a las exigencias del medio. Ésta es la lectura que cualquiera que actúe sobre los mismos debe realizar, ya que ahí se esconden las mejores lecciones que los asentamientos rurales nos proporcionan, y que debemos tener en cuenta a la hora de valorarlos y protegerlos como un patrimonio único. Ahora bien, la recuperación de esos asentamientos y su adecuación a las nuevas necesidades exigen de sus responsables un cierto grado de transformación, sometiendo al asentamiento a una evolución contenida.

\section{BIBLIOGRAFÍA}

- ACÍN FANLO, J. L.; PINILLA NAVARRO, V. (coord.) (1995) Pueblos abandonados. ¿Un mundo perdido? Zaragoza: Universidad de Zaragoza, Diputación de Huesca, 1995

- BERNARD RIVERA, P. M. (1992) Programa global para la rehabilitación de núcleos deshabitados y semideshabitados en Áreas de interés Especial del Pirineo Zaragoza. Zaragoza: DGA, 1992

- BERNAD RIVERA, P. M.; CASTELLANOS OÑATE, J. M. (1982) Pueblos deshabitados del Alto Aragón. Estudio de la comarca de Sobrarbe. Zaragoza: Colegio Oficial de Arquitectos de Aragón, 1982

- CUESTA, J. M. (2001) La despoblación del Sobrarbe. ¿Crisis demográfica o despoblación? Zaragoza: CEDDAR, 2001

- BIARgE, A.; BIARGE, F. (2000) Piedra sobre piedra. El paisaje Pirenaico humanizado. Huesca: Autoedicion, 2000

- SERRANO LACARRA, C. (coord.) (2007) Despoblación y Territorio. Zaragoza: CEDDAR, 2007

- UBieto ARTETA, A. (1985) Historia de Aragón, los pueblos y los despoblados. Zaragoza: Anubar Ediciones, 1985 